\title{
Rhinolitiasis about 16 Cases and Review of the Literature
}

\author{
Othmane Benhoumad, Mohamed Chehbouni, Youssef Rochdi, and Abdelaziz Raji
}

\section{ABSTRACT}

Rhino lithiasis is defined as a solid concretion secondary to the gradual deposition of calcium salt around an exogenous or endogenous central formation in the nasal cavity it is a relatively common condition in developing countries. We report a retrospective study spread over period of 9 years, from January 2012 to December 2020, relating to 16 cases of rhino lithiasis treated in our department. The protocol Included for each patient: an anamnesis, an ENT examination meticulous and systematic scam. Patients consulted for purulent and fetid rhinorrhea associated with nasal obstruction rhino lithiasis affected as many adults as children, the formal sex was affected in $68 \%$ of cases un favorable socioeconomic conditions were noted, in $\mathbf{7 5 \%}$ of cases diagnostic times ranged from six months to ten years, the examination she the rhinolith as a grayish, hard concretion, unilateral in 15 cases ( 5 cases on the right an on the left ) and bilateral in one case in $\mathbf{2}$ cases, a foreign body was visualized on endonasal examination, the treatment consisted of an instrumental extraction by natural means $(\mathbf{1 0 0 \%}$ of cases) under general Anas the sea in $\mathbf{7 5 \%}$ of cases the size of the rhinoliths varied between 5 to $30 \mathrm{~mm}$ in diameter. An underlying foreign body was found intraoperatively in 6 cases. Complication was rare (epistaxis: 3 cases), No recurrence was detected. It is always necessary to evoke a rhinolithiasis in front of a chronic unilateral fetid rhinnorhea with or without nasal obstruction on the same side. The nasosinus scanner confirms the diagnosis and helps to prepare the therapeutic strategy alongside the endonasal endocopic examination with the rigid optic which remains a means used both in diagnosis and in therapy.

Keywords: Rhinolithiasis, nasal rhinorrhea, nasal foreign body.
Submitted : May 06, 2021

Published : June 25, 2021

ISSN: 2593-8339

DOI: 10.24018 / ejmed.2021.3.3.867

\section{Othmane Benhoummad}

Assistant professor in ENT and cervicofacial surgery, Mohamed VI University Hospital of Marrakech, Faculty of medicine and pharmacy of Marrakech, Cadi Ayyad University, Morocco.

(e-mail: benhoummadorl@ gmail.com)

Mohamed Chehbouni *

Specialist in ENT and cervico-facial surgery, Mohamed VI University Hospital of Marrakech, Morocco.

(e-mail: dr.chehbouni@gmail.com)

Youssef Rochdi

Professor in ENT and cervico-facial surgery, Mohamed VI University Hospital of Marrakech, Professor of higher education, Faculty of Medicine and Pharmacy of Marrakech, Cadi Ayyad University, Morocco.

(e-mail: rochdi_86@hotmail.com)

Abdelaziz Raji

Head of ENT and cervico-facial surgery,

Mohamed VI University Hospital of Marrakech, Professor of higher education, Faculty of Medicine and Pharmacy of Marrakech, Cadi Ayyad University, Morocco.

(e-mail: raji.abdelaziz ${ }^{\circledR}$ gmail.com)

*Corresponding Author

\section{INTRODUCTION}

Rhino lithiasis is defined as a solid concretion secondary to the progressive deposition of calcium salts around a ex oogenus central formation, resorbable or not, of variable shape and size it is a rare affection especially in developed countries, given the frequency of foreign bodies in the nasal cavities, environment pollution, Lon sanitary and socioeconomic level as well as certain traditions (endonasal herbal medicine, ethnic customs) [1], [2].

\section{MATERIALS AND METHODS}

This is a retrospective study spanning 9 years, from January 2012 to December 2020, involving all patients with rhinolithiasis, the protocol included for each patient an anamnesis (reason for consultation, circumstances and history), a rhino logical examination aided by rhinocavoscopy after local anesthesia with 5\% naphazoline xylocaine and aspiration of nasal debris, supplemented by a nasosinus tomodensitometry. Postoperative follow-up was done after 30 days, three months, six months, twelve months, and twenty-four months.

\section{RESULT}

This is a study of 16 cases of rhino lithiasis the female sex was affected in 68 of cases. the average age 24 years with extremes ranging from 6,5 years to 67 years adverse socioeconomic condition were noted in $75 \%$ of cases all of which were of rural origin.

The diagnostic time varied between six months and ten years with an average of three and a half years. Its story found two cases of allergic rhinitis, with no notion of nasal trauma, endonasal or dental surgery chronic purulent fetid rhinorrhea and nasal obstruction (unilateral in 15 cases and bilateral in 1 case). Were the major symptoms present in all patients 
associated with epistaxis in 4 cases, anosmia in 2 cases and headache in only 1 case.

The anterior rhinoscopy supplemented by rhinocavoscopy at 0 optic after aspiration and local Anesthesia with xylocaine naphazoline $5 \%$ allow to highlight the rhinolite in the form of a grayish, hard concretion, unilateral in 15 cases ( 5 cases the right and 10 cases on the left) and bilateral in one case. In 2 cases a foreign body was visualized on endonasal examination.

The nasosinus competed tomography in axial and coronal section was systematic shooing a calcium hyper density in $100 \%$ of cases, also specialying the site and the dimensions of the rhino lithiasis without any visualized foreign body (Fig. 1).

The therapeutic management consisted of an instrumental extraction with the straight Blakeslee for ceps under endoscopic endonasal guidance by natural routes $(100 \%$ of cases under general anesthesia in $75 \%$ of the cases and under local anesthesia with xylocaine naphazoline $5 \%$ in $25 \%$ of the cases (Fig. 2).

A detailed examination of the rhinoliths after rhinoliths varied between 05 to $30 \mathrm{~mm}$ in diameter with an average of $18 \mathrm{~mm}$. an underlying foreign body was found Intraoperatively in 6 cases is $37 \%$ of cases (plant: 5 cases, shewing gum: 1 case) (Fig. 3).

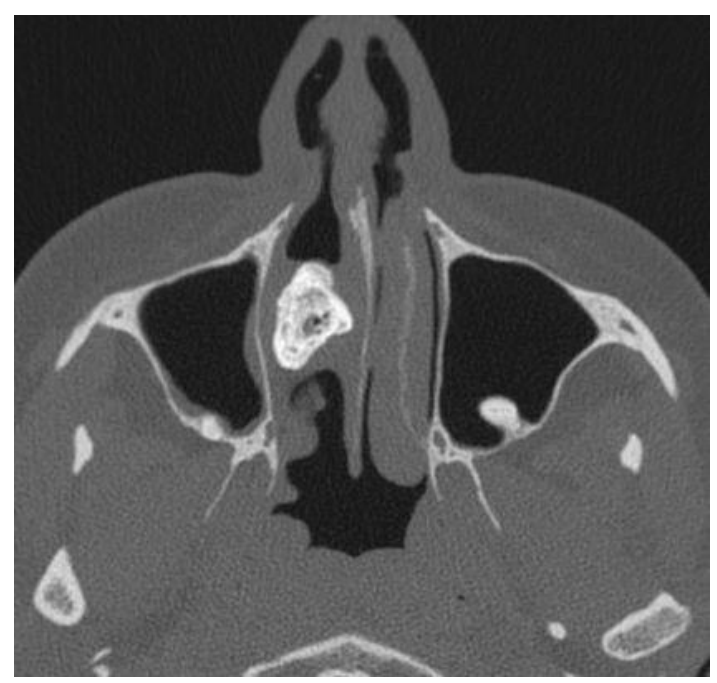

Fig. 1. Facial in axial section showing rhinolithiasis the left nasal cavity.

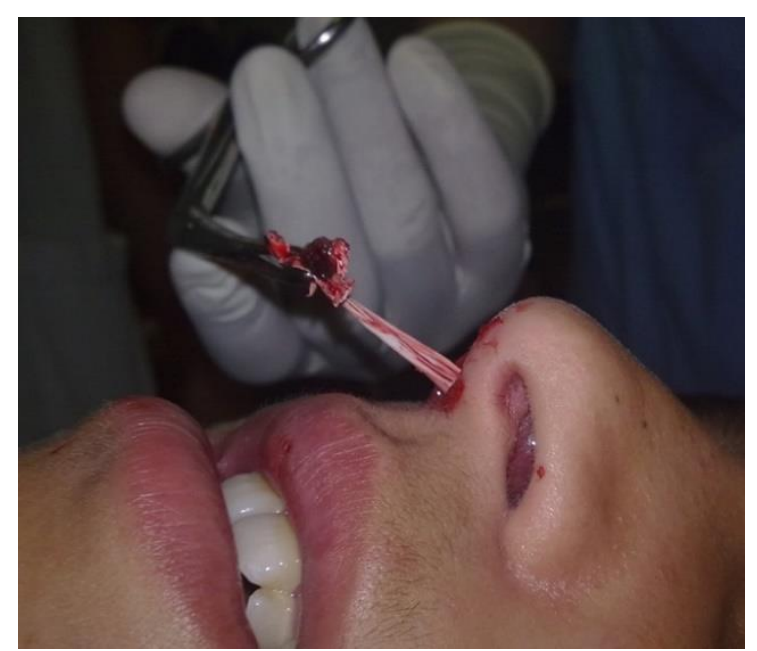

Fig. 2. Instrumental extraction with right blackishly forceps of rhino lithiasis of left nasal cavity.
Local postoperative disinfection was systematic for 7 days, postoperative outcomes were favorable in 13 patients three patients presented anterior epistaxis immediately after surgery the long-term course did not show any recurrence over an average follow of 20 months.

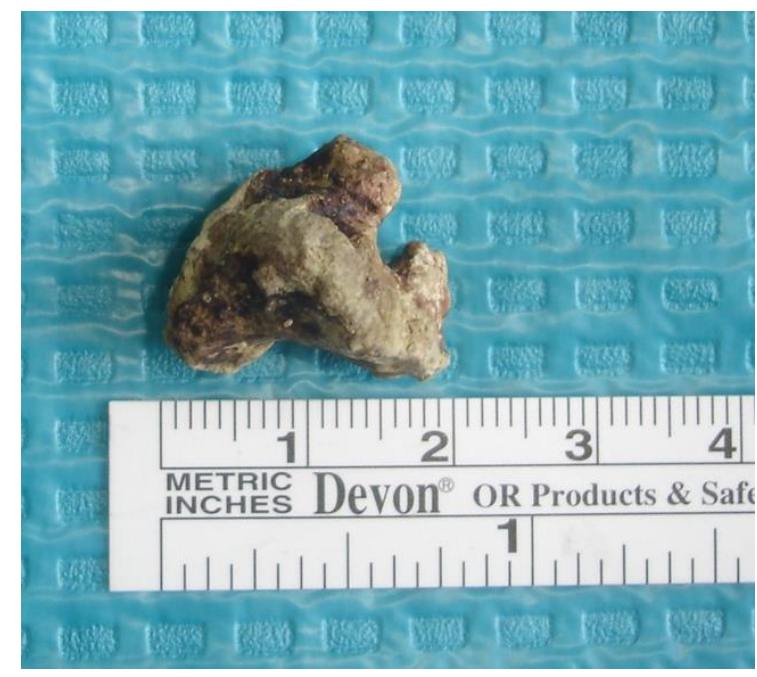

Fig. 3 Rhinolith $20 \mathrm{~mm}$ in diameter.

\section{DISCUSSION}

Rhino lithiasis is rare in the west, an estimate of 3 new cases /years / 100,000 Ha. Has been reported in the literature in developing countries. The first description dates back to 1654 by Bartholin, we can estimate the number of cases reported in the literature worldwide between 700 to 800 cases [1], [3].

The Pathogenesis of rhino lithiasis is not yet understood, in the literature, there are two different training methods. The first is an exogenous mode due to the precipitation of calcareous salts on a pre - existing foreign body (cereals, small fragment of stone, plastic parts, insect, fragment of food glass, bud, wood, fruits, grain, bud, cotton, remains of igas buffer). The second is an endogenous mode due to the precipitation of calcareous salts on a biological substance (dry secretion, blood clots, cell lysis products, mucosal nenosis, tooth fragment, dried pus, secretions from the sinus or the lacrimal al glands [1]-[3].

Rhino lithiasis is a a predominantly female condition, probably because women blow their noses weaker than men, we also note that the right side is the most frequently affected given the high number of right-handed people who put foreign bodies in the right nasal cavity, especially in children [1], [2], [4].

The clinical picture of rhinolithisis is nonspecific and is mainly characterized by purulent fetid rhinorrhea, associated in most cases with nasal obstruction [5].

Sometimes episodes of epistaxis nasopharyngitis halitosis, headaches, pain in the face are found in connection with secondary sinus infections or facial neuralgia, rare cases are revealed by lacrimation, repeated conjunctivitis or by neuropsychic disorders (isolation, agitation, depression, socio-professional difficulties) [1].

Asymptomatic forms of accidental discovery (ENT) examination, oral care examination forms of the facial mass are frequently cited in the literature. 
Exceptionally rhinoliths have been discovered intraoperatively (DCRS septoplasty, maxillary osteotomy) or in the follow-up of a patient victim of a public highway [6].

The positive diagnosis of rhinolihiasis is based mainly on the data of the examination focused on the endonasal examination (after aspiration of nasal secretions and wicking by wicks soaked with xylocaine naphazoline at 5\%) wish shows a blackish or yellow formation-greyish with an irregular surface hard to the touch, sits mainly at the level of the floor of the nasal cavity (inferior meatus or in terturbino septal space), most often halfway between the anterior and posterior part [7], [8].

The literature reports unusual localizations of rhinolithiasis; pure septal site, sinus of the face (maxilla, frontal) roof of the oral cavity after palatal perforation or unusual localization on concha bullosa [4], [7], [8].

Computed tomography the nasosinus cavities in coronal and axial section makes it possible to objectify the rhinolite in the form of an opacity of calcium density (total or partial) makes it possible to specify the site, shape, size, and presumed origin of the rhinolith (presence or not of a foreign body). Computed tomography is a great contribution to the choice of the therapeutic strategy (extraction method, surgical difficulty, choice of voice first) [1]-[5].

Rhino lithiasis spontaneously progress to worsening symptoms, in rare cases, if the rhinolith is small, spontaneous elimination maybe noted during a blowing or sneezing [1], [2], [6], [8].

Rhino lithiasis pose a problem of differential diagnosis with all nasal sinus pathologies with calcium density: benign tumors (osteoma), malignant tumors (chondrosarcoma, osteosarcoma), bone sequesters from osteomyelitis or radionecroisis syphilis or tuberculosis with calcification [1], [2], [9], [10].

The therapeutic management of rhinolithiasis consists of nasal extraction under endoscopic control (rigid optics) [9], [12]. the procedure is performed under local anesthesia or general anesthesia depending on the teams [1]-[8]. other authors reserve general anesthesia for special cases (child, non-cooperative patient) with orotracheal intubation to protect the upper airways from the risk of rhinolithiasis falling [1].

The gesture consists of extraction from back to front with a suitable hook, extraction with the straight Blakrsley forceps or rarely by suction, the placement of a silastic blade in contact with the nasal septum for 15 days may be necessary in case of traumatic and repetitive maneuvers, to prevent nasal synechiae [11]-[13].

External extraction (sub-labial rhinotomy, degloving, etc.) of rhinolithiasis is exceptional with specific indication (large rhinolite, incarcerated, vestibular or turbinoseptal pathologies, massive granulomatous reaction, associated lesions)

A detailed analysis is made after extraction of the rhinolith (size, weight, presence of foreign body) [1].

The postoperative consequences are generally simple, and recurrences are rare.

\section{CONCLUSION}

Rhinolithiasis is defined as a solid concretion secondary to the gradual deposition of calcium salts around an exogenous or endogenous central formation, rare in the west, and is clinically revealed by purulent fetid rhinorrhea, associated with nasal obstruction. The positive diagnosis of rhinolithiasis is mainly based on clinical examination. The contribution of nasosinus CT is essential for the therapeutic stategy,which consists of endonasal extraction under endoscopic control.

\section{REFERENCES}

[1] Kharoubi S. Revue générale sur les rhinolithiases. Annales d'oto-rhinolaryngologie et chirurgie cervico-faciale 125 (2008) 11-17.

[2] Merol JC, Schmidt P, Legros M, Chays A. Corps étrangers des fosses nasales Rhinolithiase, Encyclopédie Médico-Chirurgicale 20-390-A10.

[3] Hadi U, Ghossaini S, Zaytoun G. Rhinolithiasis: A forgotten entity. Otolaryngol Head Neck Surg 2002; 126:48-51.

[4] Orhan K, Kocyigit D, Kisnisci R, Paksoy CS. Rhinolithiasis: An uncommon entity of the nasal cavity. Oral Surg Oral Med Oral Pathol Oral Radiol Endod 2006;101: e28-32.

[5] Sumbullu MA, Tozoglu U, Yoruk O, Yilmaz AB, Ucuncu H Rhinolithiasis: the importance of flat panel detector-based cone beam computed tomography in diagnosis and treatment. Oral Surg Oral Med Oral Pathol Oral Radiol Endod 2009;107: e65-7.

[6] ] Al Yaghchi C, Marais J. Rhinolithiasis: Long-Term Complication of Maxillary Osteotomy. J Oral Maxillofac Surg, 67:1745-1746, 2009.

[7] Keck T, Liener K, Strater J, Rozsasi A. Rhinolith of the nasal septum. International Journal of Pediatric Otorhinolaryngology 53 (2000) 225 228.

[8] Pinto LS, Campagnoli EB, de Souza Azevedo R, Lopes MA, Jorge J. Rhinoliths causing palatal perforation: case report and literature revie. Oral Surg Oral Med Oral Pathol Oral Radiol Endod 2007;104: e42-6.

[9] Munoz A, Pedrosa I, Villafruela M. "Eraseroma"as a cause of rhinolith:CT and MRI in a child. Neuroradiology (1997) 39: 824-826.

[10] Aksungur EH, Binokay FB, Biçakçi $\mathrm{K}$ et al. A rhinolith which is mimicking a nasal benign tumor. European Journal of Radiology 31 (1999) 53-55.

[11] Bayram B, Deniz K, Polat Ozsoy O, Uckan S. A Simple Surgical Approach for Management of the Rhinoliths: Case Report. J Oral Maxillofac Surg 2011 May;69(5):1403-7.

[12] Yuca K, Caksen H, Etlik O. The importance of rigid nasal endoscopy in the diagnosis and treatment of rhinolithiasis. Auris Nasus Larynx 33 (2006) 19-22.

[13] Şenkal HA, Suslu HE, Unal OF. A rare cause of rhinolithiasis: Ectopic tooth. International Journal of Pediatric Otorhinolaryngology Extra (2006) 1, 249-252.

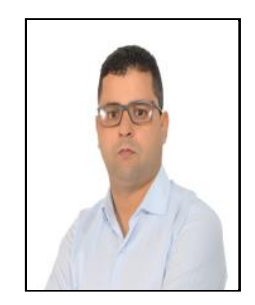

Benhoummad Othmane

Place and date of birth: Safi, Morocco 24-07-1982. Doctor of medicine, assistant professor in ENT surgery.

Marrakech Faculty of Medicine and Pharmacy Cady Ayyad University Marrakech, Morocco. 